\title{
Editorial
}

\section{The paths to death and differentiation}

\author{
AJ Levine ${ }^{\star 1}$ \\ Cell Death and Differentiation (2011) 18, 1391-1392; doi:10.1038/cdd.2011.41
}

Ever since evolution began experimenting with the creation of invertebrate multicellular organisms, cell death and terminal differentiation have been employed as a central set of processes to permit these organisms to develop, survive and to reproduce. Cell death and differentiation are employed in several related processes: (1) the development of the organism, (2) the asexual regeneration of the organism from stem cells after it is wounded or dissociated into its cellular parts, (3) the death and regeneration of cellular and tissue components over the normal lifespan of the organism (tissue turnover), (4) the sexual reproduction of an organism through its primordial germ cells and (5) cell death and differentiation are employed as processes to limit pathogenesis in an organism.

A terrific example of the cooperation of cell death and differentiation in the development of an organism comes from the study of $\mathrm{C}$. elegans where a fertilized egg gives rise to an organism with 959 cells in the hemaphrodite derived from many developmental lineages where 131 cells reproducibly die by the genetically programmed processes of apoptosis. In the central nervous system of humans a programmed developmental production of neurons is further shaped by environmental experiences, which reinforce neuronal connections and neuronal cell death when such connections are not made. The asexual regeneration of an organism or parts of an organism has been studied in worms, fish and amphibians and is regulated by the induction or reprogramming of stem cells that can regenerate parts or entire organisms. How these latent stem cells are formed and regulated so as to not produce developmental abnormalities or cancers until an appropriate stress signal is given remains a mystery. Starting with the vertebrates, (fish) tissue regeneration and turnover throughout the life span of the organism requires coordination between death and differentiation that permits the organism to function efficiently for longer periods of time extending its lifespan. This in turn permits more complex organisms with longer gestation periods, longer times to develop to sexual maturity and enough time to have smaller numbers of offspring that are well taken care of for longer periods. From hydra to humans, death and differentiation have important roles in the surveillance of fidelity of the germ line. A cell death program in germ cells is activated by DNA damage, starvation conditions and many other stresses all across the invertebrate and vertebrate phyla. The primordial germ cells harbor the ultimate information for immortality, the expression of which resides in a program of differentiation producing two (or more) sexes that enhance the exchange of combinations of information. The passage of faithful information is insured by death in lineages that harbor too many mistakes.

Cell death and differentiation are employed effectively to limit pathogenesis. This is observed in both the innate immune system of the invertebrates and vertebrates and in the adaptive immune system of vertebrates. Programmed cell death in response to infection limits replication and spread of an organism. Differentiation programs are commonly activated in response to infections or cytokines induced by infections. The origins and propagation of cancers are responded to by programs for cell death, cell senescence and terminal differentiation. Tumor necrosis factor, trail, fas and $\mathrm{p} 53$ respond to oncogene activation and abnormal developmental pathways triggered by oncogene and tumor suppressor gene mutations. The p53 protein dramatically lowers the efficiency of producing induced pluripotent stem cells in culture. Here p53 impacts upon dedifferentiation processes and epigenetic changes by limiting cell division or activating cell death. In breast cancers there is a close association of a stem cell phenotype with p53 mutations in a tumor type. Here the $\mathrm{p} 53$ protein, which can start the process of programmed cell death is also regulating the epigenetic changes in a cell and developmental fates of these cells. A large number of disorders can arise by the failure of components of the death and differentiation pathways. Neurodegenerative disorders are a tragic example of such processes. Indeed the lifespan of an organism may be determined by the regulation of stem cell lineages over a lifetime. Here decisions of renewal, differentiation and death may change the balance between long life and a brief existence.

The reviews that follow this introduction deal with many of these issues employing specific examples of programmed cell death $^{1}$ or escape from death ${ }^{2,3}$ and discussing the mechanisms that regulate them. The regulation of the ubiquitin ligases ${ }^{4}$ and caspases $^{5}$ are at the center of the death and differentiation pathways. The p53 pathway can lead to apoptosis, senescence cellular repair processes and through its target gene, Wig-1, stem cell fates. ${ }^{6}$ Similarly the PML

\footnotetext{
${ }^{1}$ Simons Center for Systems Biology, Institute for Advanced Study, Princeton, NJ 08540, USA

*Corresponding author: AJ Levine, Simons Center for Systems Biology, Institute for Advanced Study, Princeton, NJ 08540, USA. Tel: + 609 734 8005; Fax: 609 924 7592; E-mail: alevine@ias.edu
} 
tumor suppressor can regulate communication between the endoplasmic reticulum and mitochondria and in doing so control an apoptotic pathway. ${ }^{7}$ The role of cell death in pathogenesis is nowhere more dramatic than in neurodegenerative disorders such as Parkinson's disease ${ }^{8}$ or ataxiatelangiectasia, ${ }^{9}$ which are reviewed in a new light in this issue. These reviews employ specific examples to cover the broader landscape of how death and differentiation combine to control pathogenesis and just what happens when these processes fail. For each of these failures we must learn the road to repair and treatment ${ }^{10}$ and we will only do that by understanding how the pathways to death function in many diverse cell types and organisms.

\section{Conflict of interest}

The author declares no conflict of interest.

1. Geering B, H-U. Cell Death Differ 2011; 18: 1457-1469.

2. Vitale I et al. Cell Death Differ 2010; 18: 1403-1413.

3. Kelly P, Strasser A. Cell Death Differ 2011; 18: 1414-1424.

4. de Bie P, Ciechanover A. Cell Death Differ 2011; 18 : 1393-1402.

5. Olsson M, Zhivotovsky B. Cell Death Differ 2011; 18 : 1441-1449.

6. Vilborg A et al. Cell Death Differ 2011; 18: 1434-1440.

7. Pinton P, Giorgi C, Pandolfi PP. Cell Death Differ 2011; 18: 1450-1456.

8. Steiner JA, Angot E, Brundin P. Cell Death Differ 2011; 18: 1425-1433.

9. Devgan SS et al. Cell Death Differ 2011; 18: 1500-1506.

10. Royer C, Lu X. Cell Death Differ 2011; 18: 1470-1477. 